\title{
Tecnologías para ahorrar agua en el cultivo de arroz
}

\author{
Technologies for water saving in rice
}

Mauricio González B. ${ }^{1}$, Ana Milena Alonso²

\section{Resumen}

El uso eficiente del agua determina el futuro del cultivo de arroz en Colombia frente a los tratados de libre comercio, ya que es un factor que condiciona el área sembrada, el rendimiento de la cosecha y los costos de producción, además de su sostenibilidad en el tiempo. Por lo tanto, se presenta una revisión de las tecnologías desarrolladas a nivel mundial enfocadas al ahorro del agua en el cultivo de arroz en condiciones aeróbicas y anaeróbicas. Se resaltan tecnologías alternativas que superan al sistema convencional en rendimiento y en la cantidad de arroz cosechado por metro cúbico de agua invertido. En la reflexión para Colombia, se destacan aspectos importantes para la construcción de la agenda de investigación y la apropiación social de las tecnologías alternativas con miras a la optimización del recurso hídrico en el cultivo de arroz; se recomienda priorizar el arroz aeróbico y la medición efectiva del consumo de agua, indispensable para controlar su uso, planear, dirigir y entender cómo estas tecnologías alternativas conducen a la recuperación de la inversión de los agricultores y a la rentabilidad del cultivo.

Palabras clave: Arroz, tecnologías, uso eficiente del agua, aeróbico, campo inundado.

\section{Abstract}

Water use efficiency, given that it conditions the sown areas, yield and production costs, as well as its sustainability, determines the future of rice production in Colombia against the FDA. This paper presents a review of the globally developed technologies for water saving in rice production under aerobic and anaerobic conditions. The authors highlight alternative technologies that surpass the conventional system in terms of yield and harvest per cubic meter of water used. In the reflection for Colombia, the authors highlight important features for the research agenda and the social appropriation of alternative technologies with a view to optimizing water resources usage for rice production. Recommendations include the prioritizing of aerobic rice and the effective measurement of water consumption, which is indispensable for its use, and for planning, directing, and understanding how these alternative technologies can lead to the recovery of the investment made by agricultural workers and crop profitability.

Keywords: Rice, Technologies, Efficient Water Use, Aerobic, Flooded Field. 


\section{Introducción}

El arroz (Oryza sativa L) es básico en la canasta familiar para cerca de la mitad de la población mundial (1), el segundo cereal con mayor área cosechada (2) y un cultivo que ejerce una gran presión sobre el recurso hídrico (PSRH; (3)). El sector agrícola en Colombia demanda anualmente 54\% del agua consumida (19386 millones (M) de $\mathrm{m}^{3}$ ), de los cuales $4185 \mathrm{Mm}^{3}$ son implementados para el cultivo de arroz y de estos, se estima que solo $962 \mathrm{Mm}^{3}$ son consumidos de forma efectiva (4). Para Tolima, como principal productor de arroz del país, la Federación Nacional de Arroceros (FEDEARROZ) y las asociaciones de usuarios de los distritos de riego (4), estiman que la demanda de agua en zonas inundables oscila entre 16000 y $30000 \mathrm{~m}^{3} /$ ha-cosecha. No obstante, el uso del modelo "Aquacrop" desarrollado por la FAO predice que la aplicación de riego debe ser de 4800 $\mathrm{m}^{3} /$ ha-cosecha (5), por lo tanto, gran parte del agua retorna al sistema hídrico con variaciones en su calidad.

El consumo de grandes volúmenes de agua en el cultivo de arrozen Colombia se asocia directamente a las malas prácticas culturales (MPC) en torno al manejo del agua y a la ineficiencia del riego por gravedad (IRG; (1)). Por su parte, la oferta hídrica se ha visto afectada por la degradación de las cuencas de los distritos de riego (DCDR) y por el cambio climático (CC), de esta forma la localización geográfica y la orografía que ubican a Colombia entre los países con mayor riqueza hídrica, no es suficiente para evitar PSRH.

Una alta PSRH está relacionada con un índice de uso de aguas (IUA) mayor a 0.5 , que corresponde a la relación entre la demanda y la oferta hídrica disponible para la misma unidad espacial de referencia en un cierto periodo de tiempo (ec. 1). En el Tolima el $68 \%$ de los municipios (32) presentan un IUA mayor a 0.5 , donde el $81.5 \%$ de agua es consumida por el sector agrícola y la mayor demanda hídrica está concentrada alrededor de los distritos de riego para el cultivo de arroz (6). Es importante tener en cuenta que el IUA se afecta por: a) la falta de educación ambiental (EA); b) la deficiente aplicación normativa (DAN); c) la poca adopción de tecnologias (AT); d) la contaminación hidrica $(\mathrm{CH})$, y e) la deforestación y la ampliación de la frontera agrícola y pecuaria (DAFAP).

$$
\mathrm{IUA}=\frac{\text { Demanda hídrica }(\mathrm{EA}+\mathrm{DAN}+\mathrm{AT}+\mathrm{IRG})}{\text { oferta hídrica }(\mathrm{DAFAP}+\mathrm{CH}+\mathrm{MPC}+\mathrm{CC}+\mathrm{DCDR})}
$$

La PSRH tiene efectos sobre el precio del arroz, ya que influye en el área sembrada, en el rendimiento y la oferta del producto. La rebaja drástica de la disponibilidad de agua en los últimos ańos es la razón por la cual se ha reducido el área sembrada de arroz de riego (Zona centro (6)) y ha aumentado el arroz secano en Colombia (Zona llanos; ver Figura 1).

El exceso de agua y la desnivelación del área cultivable generan erosión, daños en la estructura física y pérdidas de suelo agrícola, aumentando el contenido de arenas de poca retención hídrica, inertes desde el punto de vista químico, carentes de propiedades coloidales y de reserva de nutrientes. Por lo tanto, con la erosión se deteriora la fertilidad y aumenta la demanda de fertilizantes. En las Figuras 2 y 3 se observa cómo el rendimiento del Arroz Paddy Seco en Colombia ha disminuido en el tiempo. El exceso de agua inhibe la germinación y el uso de láminas muy altas y tempranas, disminuyen el macollamiento y alargan el tallo de las plántulas favoreciendo su volcamiento (7). Todo lo anterior resulta en incremento de costos por mayor uso de semilla, fertilizantes y agua; y además, por la necesidad de remoción de sedimentos presentes en los canales que aumentan la tarifa cobrada por los distritos de riego.

El problema ambiental y de rentabilidad del cultivo de arroz mencionado anteriormente reafirma la necesidad de implementar tecnologías alternativas que contribuyan al uso eficiente del recurso hídrico, lo cual se ha ratificado en las diferentes agendas prospectivas y de desarrollo tecnológico del Gobierno Colombiano (Plan Nacional de Desarrollo 2010-2014, 2014-2018; Visión 
Colombia II Centenario: 2019). Es perentorio que los productores de arroz se preparen para incrementar eficazy eficientementela productividad de arroz, ya que en el 2030 el tratado de libre comercio permitirá la importación de arroz desde Estados Unidos (EUA) sin costo de arancel.

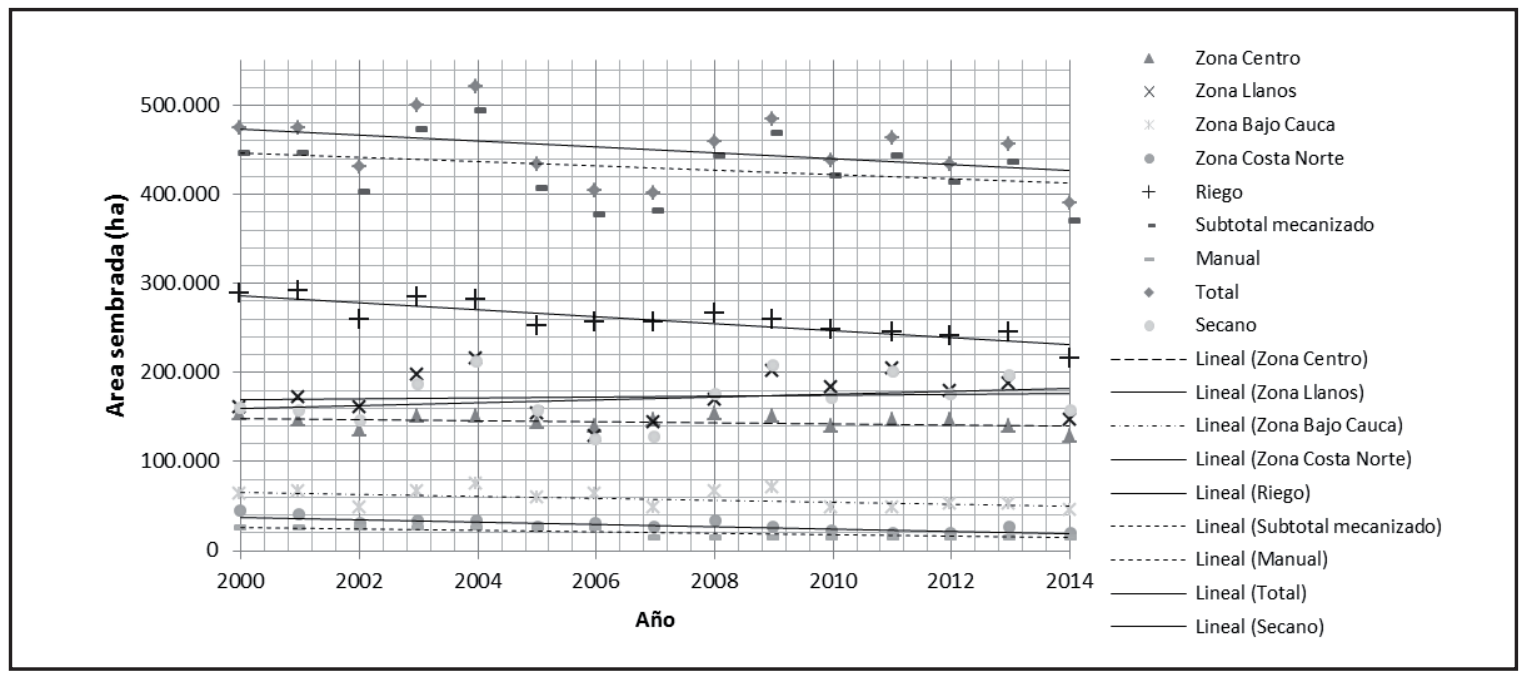

Figura 1. Superficie sembrada de arroz en Colombia por sistema y zona de producción (Fuente: 8).

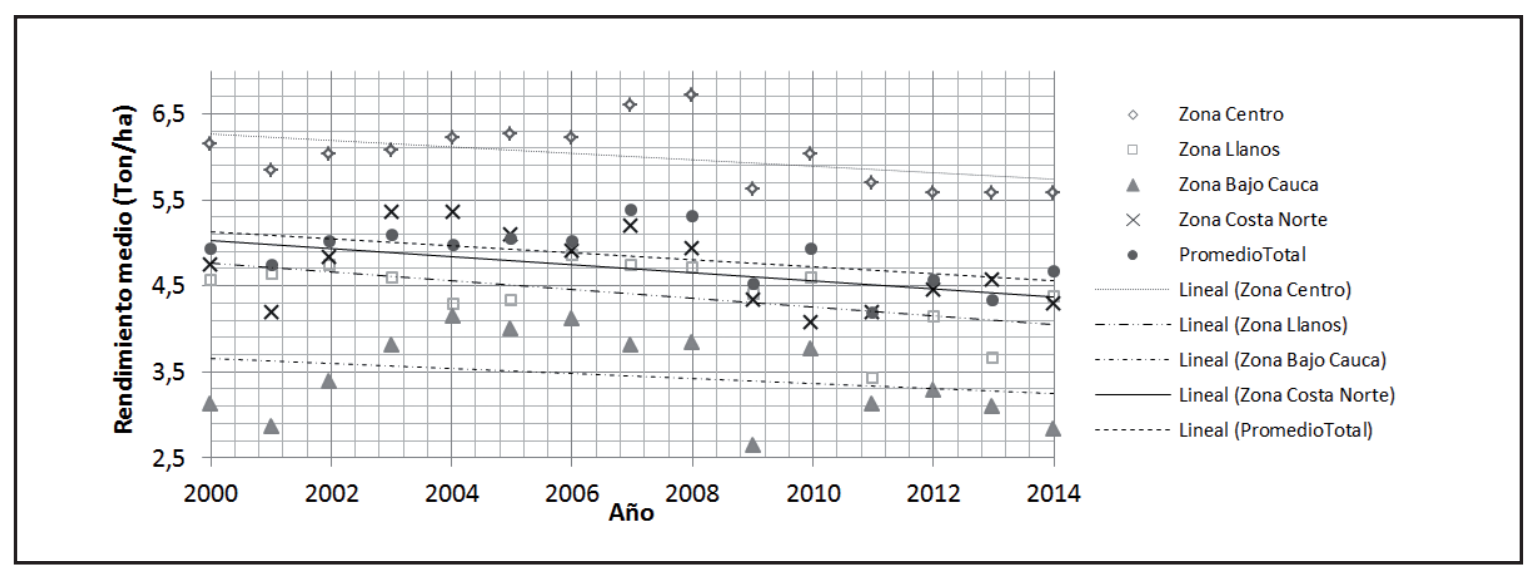

Figura 2. Rendimiento anual de arroz paddy seco en Colombia por zonas (Fuente: 8).

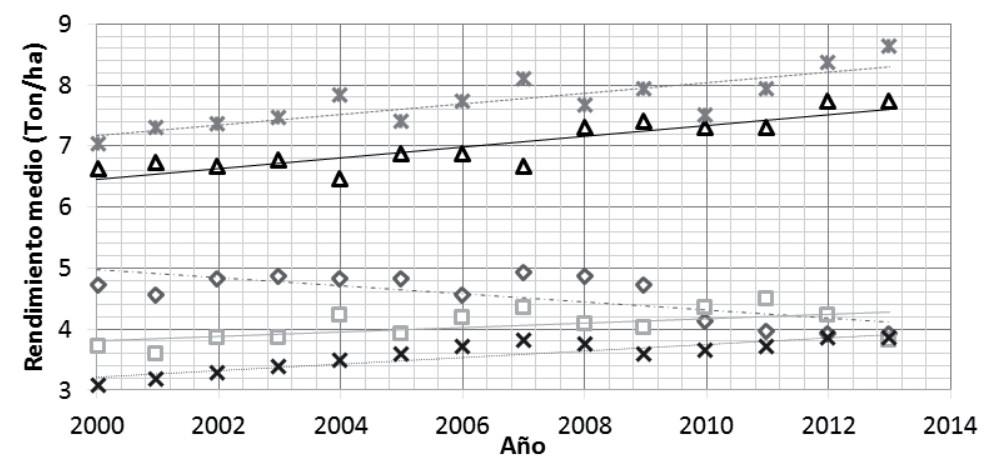

- Colombia

ㅁcuador

$\Delta \quad$ Peru

$\times \quad$ Filipinas

× EUA

Lineal (Colombia)

Lineal (Ecuador)

Lineal (Peru)

Lineal (Filipinas)

Figura 3. Rendimiento anual de arroz paddy promedio en Colombia y en otros países (Fuente: 9). 
Tecnologías disponibles para la optimización del recurso hidrico

En cuanto al arroz aeróbico, se destaca el arroz bajo riego por aspersión. En la transición de arroz aeróbico a arroz en condiciones anaeróbicas, se encuentra el sistema intensivo del cultivo del arroz (SICA), el sistema de producción de arroz con suelo cubierto (Ground Cover Rice Production System, GCRPS), el arroz cultivado en Suelo Saturado (SS) o con intermitencia en el riego (IR), donde se pueden utilizar tecnologías para alternar la humedad y el secado (Alternate Wetting Drying, AWD), politubos con múltiple entrada de agua (Multiple Inlet Rice Rrrigation, MIRI) e hidroretenedores, además de técnicas para la eficacia del riego inicial y para la fertilización nitrogenada. En el arroz bajo inundación, se resaltan técnicas de riego con: 1) diques rectos (DR); 2) pendiente cero en el terreno; 3) diques en contorno a curva nivel (DC); y 4) riego corrido diseñado con contornos bajo la experticia del operario. En la concesión de la agricultura como un proveedor de servicios ecosistémicos, aparece la agroforestería, la rizipiscicultura y la agroecología.

A partir de la revisión bibliográfica, la información fue sintetizada mediante gráficas (presentadas en las Figuras 4 y5), basadas en la Tabla 1, 2 y 3, que comparan los rendimientos (R) y los coeficientes de uso eficiente del agua (Water Use Efficiency, WUE; ec. 2) para el arroz cultivado con tecnologías alternativas (T.A) y el sistema convencional de arroz inundado (SC). En ellas, se resaltó la zona tropical (T) y subtropical (ST) donde se realizó el estudio, ya que el arroz cultivado en el trópico tiene características diferentes a las del subtrópico (1). El ahorro de agua (AA) fue calculado según la ec. 3 .

WUE $=\mathrm{Kg}$ arroz/agua total aplicada durante la producción $\left(\mathrm{m}^{3}\right)$

$\mathrm{AA}=\left(\mathrm{WUE}_{\mathrm{TAA}}-\mathrm{WUE}_{\mathrm{SC}}\right) / \mathrm{WUE}_{\mathrm{SC}} \mathrm{x} 100$

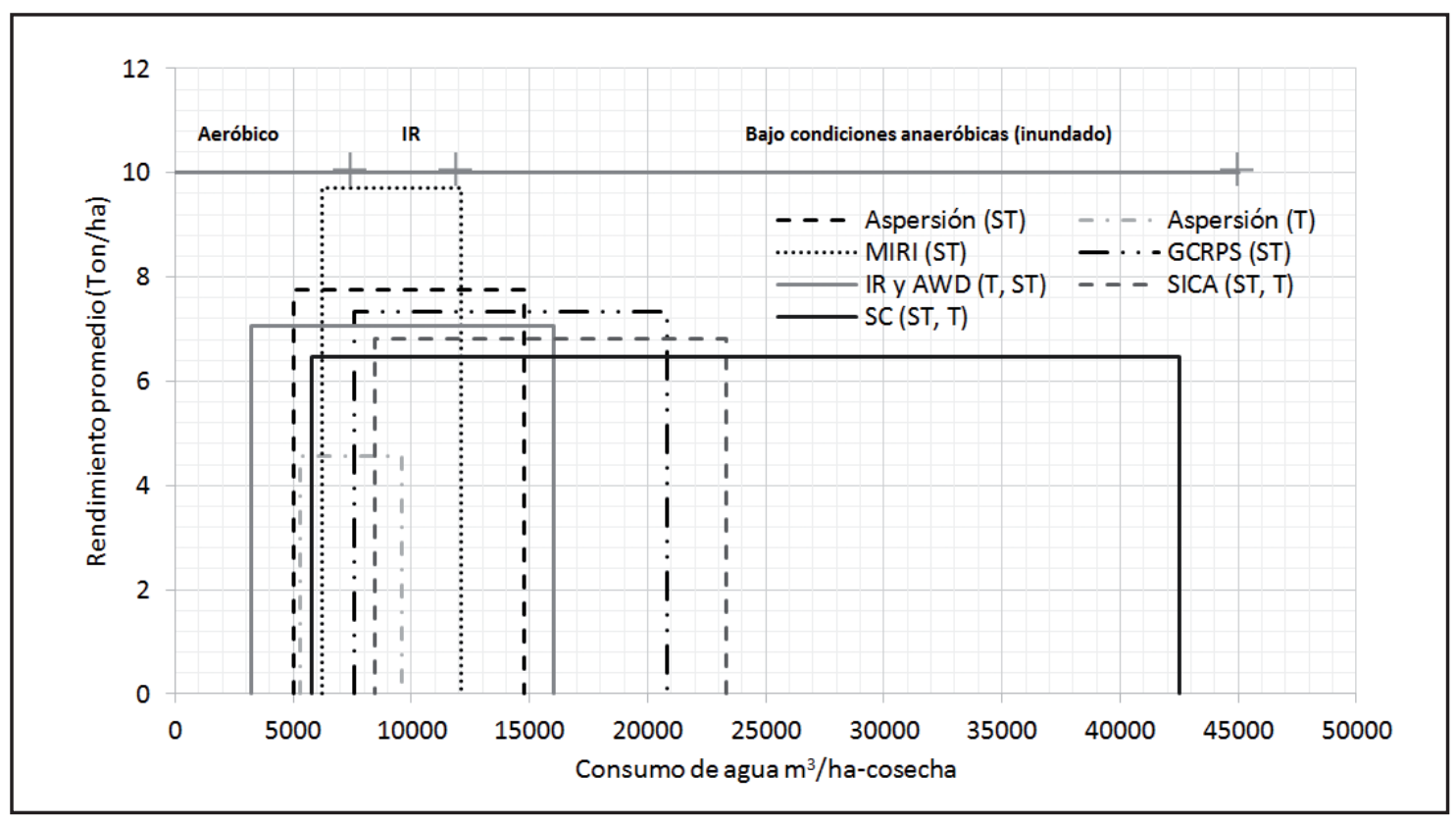

Figura 4. Rendimiento promedio y rango de consumo de agua en el cultivo del arroz con diferentes tecnologías. Fuente: Autores 
Las investigaciones resaltan que el control adecuado de malas hierbas y la aplicación mínima de agua (5000 $\mathrm{m}^{3} /$ ha-cosecha), son aspectos clave para la producción de arroz por aspersión (ver Figura 4). El pivote central es una de las tecnologías que logra la mayor productividad del agua con un promedio de $1.44 \mathrm{~kg} / \mathrm{m}^{3}$ superando al SC. De acuerdo con FEDEARROZ (10), un pivote central de 400 metros de longitud en un cultivo que demanda $6.4 \mathrm{~mm} /$ día, riega 50 ha con un intervalo de riego de 4 días, 16 horas de riego diario, un hidro-módulo de 1.31t/s/ha, un caudal de $65.10 \mathrm{l} / \mathrm{s}$ y una potencia de $75 \mathrm{Kw}$. Como aspectos importantes a tener en cuenta, el pivote central se adapta a topografías onduladas en cualquier edad del cultivo, reduce los costos operativos del riego tradicional por inundación, permite la aplicación eficiente de fertilizantes y químicos con uniformidad, y se estima que tiene una vida útil promedio de 20 años.

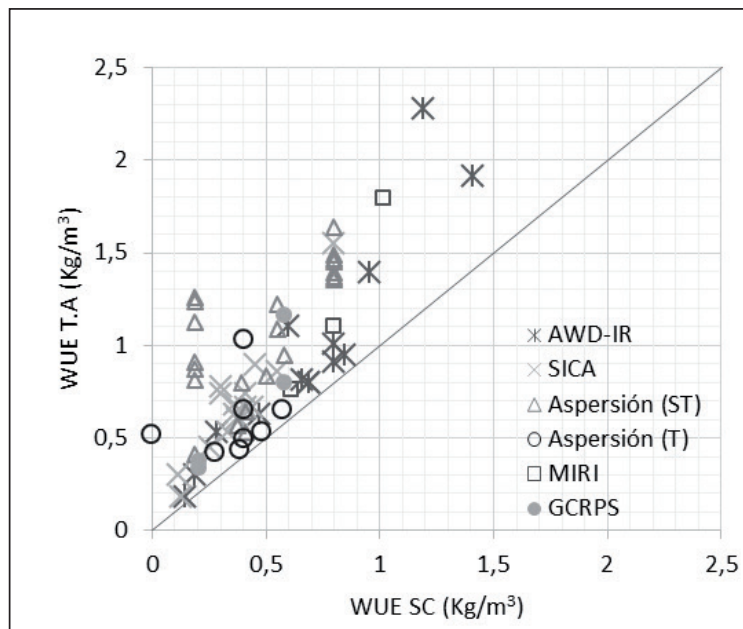

a)

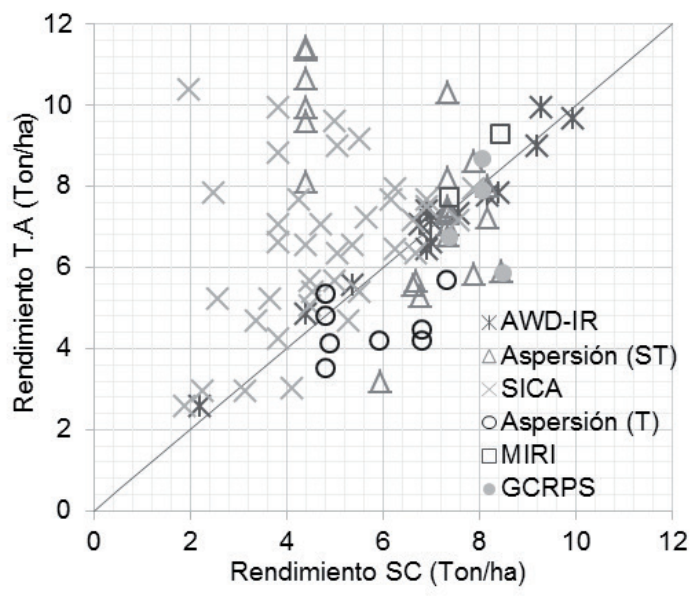

b)

Figura 5. Características de la producción de arroz con tecnologías para el ahorro de agua vs el sistema convencional. Gráfico P-P de dispersión con: a) coeficientes WUE y b) rendimientos. Fuente: Autores.

La tecnología SICA fomenta el trasplante de plantas no mayores a veinte días de germinadas, el empleo de una postura por nido, además de la implementación del fangueo (enlodar) para disminuir las cantidades requeridas de agua para riego con un excelente rendimiento (AA y WUE de $83 \%$ y $0.67 \mathrm{Kg} / \mathrm{m}^{3}$; ver Figura 2). En Colombia, en el municipio de Purificación-Tolima, SICA produjo un $15.8 \%$ más de arroz por hectárea que en el SC (11).

La tecnología GCRPS, implementada en los países del subtrópico, consiste en cubrir el suelo con una delgada capa de plástico y solo cuando el contenido de agua en el suelo cae por debajo de un valor determinado se aplica riego (12). Este sistema ayuda a conservar el calor, aliviar el estrés por las bajas temperaturas, reducir la evaporación y la filtración en la etapa de crecimiento, obteniendo en promedio un AA y WUE de $67.3 \%$ y $0.66 \mathrm{~kg} / \mathrm{m}^{3}$, respectivamente. La GCRPS también disminuye la contaminación ambiental, debido a la escasa aplicación de herbicidas debido a la película de plástico que impide la germinación y el desarrollo de arvenses, y es prometedor para la reducción de las emisiones de gases de efecto invernadero (13). 
Tabla 1. Consumo de agua en arroz con tecnologías alternativas (T.A) y el Sistema Convencional (SC) en el trópico (Fuente: Autores).

\begin{tabular}{|c|c|c|c|c|c|c|c|c|c|}
\hline \multirow{2}{*}{ País } & T.A & SC & R. T.A & R. SC & \multirow{2}{*}{$\begin{array}{c}\text { A. A } \\
\%\end{array}$} & WUE T.A & WUE SC & \multirow{2}{*}{ Fuente } & \multirow{2}{*}{ T.A } \\
\hline & \multicolumn{2}{|c|}{ m3/ha-cosecha } & \multicolumn{2}{|c|}{ Ton/ha } & & $\mathrm{Kg} / \mathrm{m} 3$ & $\mathrm{Kg} / \mathrm{m} 3$ & & \\
\hline \multirow{5}{*}{ Filipinas } & 5818 & 11500 & 6.4 & 6.9 & 83.3 & 1.10 & 0.60 & (14) & AWD \\
\hline & 15160 & 28750 & 7.8 & 8.4 & 79.3 & 0.52 & 0.29 & \multirow{4}{*}{$(15)$} & \multirow{4}{*}{ SS } \\
\hline & 3910 & 5770 & 7.7 & 8.2 & 33.8 & 1.90 & 1.42 & & \\
\hline & 4770 & 7280 & 6.6 & 7.0 & 44.8 & 1.39 & 0.96 & & \\
\hline & 3240 & 6310 & 7.3 & 7.6 & 89.2 & 2.27 & 1.20 & & \\
\hline \multirow{2}{*}{ Cuba } & \multirow{2}{*}{9039} & \multirow{2}{*}{$12339^{*}$} & 7.01 & 3.8 & 151.8 & 0.78 & 0.31 & $(16,17)$ & \multirow{10}{*}{ SICA } \\
\hline & & & 6.6 & 3.8 & 137.1 & 0.73 & 0.31 & (18) & \\
\hline Colombia & 8958 & $12491^{*}$ & 7.5 & 6.9 & 51.6 & 0.84 & 0.55 & $\left(11,19^{*}\right)$ & \\
\hline Kenia & 9610 & 10741 & 14.8 & 8.7 & 90.1 & 1.54 & 0.81 & $(20)$ & \\
\hline \multirow{6}{*}{ India } & 9846 & 14318 & 6.4 & 6.3 & 47.7 & 0.65 & 0.44 & $(21)$ & \\
\hline & 9574 & 12222 & 6.5 & 4.4 & 88.9 & 0.68 & 0.36 & (22) & \\
\hline & 10566 & 13636 & 5.6 & 4.5 & 60.6 & 0.53 & 0.33 & (23) & \\
\hline & 10955 & 13476 & 7.2 & 5.7 & 57.1 & 0.66 & 0.42 & $(24)$ & \\
\hline & 9180 & 13889 & 5.6 & 5.0 & 69.4 & 0.61 & 0.36 & $(25)$ & \\
\hline & 8514 & 12439 & 6.3 & 5.1 & 80.5 & 0.74 & 0.41 & (26) & \\
\hline \multirow{3}{*}{ India } & 7340 & \multirow{3}{*}{12000} & 4.8 & & 65.0 & 0.66 & & \multirow{2}{*}{ (27) } & \multirow{8}{*}{ Aspersión } \\
\hline & 5300 & & 5.4 & 4.8 & 160.0 & 1.04 & 0.40 & & \\
\hline & 7000 & & 3.5 & & 25.0 & 0.50 & & $(28)$ & \\
\hline \multirow{3}{*}{ Filipinas } & 9545 & 15526 & 4.2 & 5.9 & 15.8 & 0.44 & 0.38 & (29) & \\
\hline & 8520 & 12750 & 5.7 & 7.3 & 15.8 & 0.66 & 0.57 & \multirow{2}{*}{ (15) } & \\
\hline & 9590 & 17590 & 4.1 & 4.87 & 53.6 & 0.43 & 0.28 & & \\
\hline \multirow{2}{*}{ Brasil } & 8495 & \multirow[t]{2}{*}{14300} & 4.5 & 6.75 & 17.0 & 0.55 & \multirow{2}{*}{0.47} & \multirow{2}{*}{$(30)$} & \\
\hline & 7605 & & 4.2 & 6.75 & 12.8 & 0.53 & & & \\
\hline
\end{tabular}

Tabla 2. Consumo de agua en arroz con tecnologías alternativas (T.A) y el Sistema Convencional (SC) en el sub-trópico (Fuente: Autores).

\begin{tabular}{|c|c|c|c|c|c|c|c|c|c|}
\hline \multirow{2}{*}{ País } & T.A & SC & R. T.A & R. SC & A. A & WUE T.A & WUE SC & \multirow{2}{*}{ Fuente } & \multirow{2}{*}{ T.A } \\
\hline & \multicolumn{2}{|c|}{ m3/ha-cosecha } & \multicolumn{2}{|c|}{ Ton/ha } & $\%$ & $\mathrm{Kg} / \mathrm{m} 3$ & $\mathrm{Kg} / \mathrm{m} 3$ & & \\
\hline \multirow{2}{*}{ India } & 23333 & 29231 & 4.2 & 3.8 & 38.5 & 0.18 & 0.13 & $(31)$ & \multirow{5}{*}{ SICA } \\
\hline & 8475 & 11538 & 5.0 & 4.5 & 51.3 & 0.59 & 0.39 & $(32)$ & \\
\hline Irak & 17931 & 33636 & 5.2 & 3.7 & 163.6 & 0.29 & 0.11 & (33) & \\
\hline \multirow{6}{*}{ China } & 14533 & 21520 & 6.5 & 5.4 & 80.0 & 0.45 & 0.25 & $(34)$ & \\
\hline & 8977 & 13696 & 7.9 & 6.3 & 91.3 & 0.88 & 0.46 & $(35)$ & \\
\hline & 15890 & 40410 & 5.9 & 8.5 & 75.9 & 0.37 & 0.21 & \multirow{2}{*}{ (12) } & \multirow{3}{*}{ GCRPS $_{\text {sat }}$} \\
\hline & 20830 & 34860 & 6.8 & 7.4 & 53.8 & 0.33 & 0.21 & & \\
\hline & 9820 & 13910 & 7.9 & 8.0 & 39.9 & 0.80 & 0.58 & \multirow{2}{*}{ (13) } & \\
\hline & 7570 & 13910 & 8.7 & 8.0 & 99.8 & 1.15 & 0.58 & & GCRPS $_{80 \%}$ \\
\hline
\end{tabular}




\begin{tabular}{|c|c|c|c|c|c|c|c|c|c|}
\hline \multirow{2}{*}{ País } & T.A & SC & R. T.A & R. SC & \multirow{2}{*}{$\frac{\text { A. A }}{\%}$} & WUE T.A & WUE SC & \multirow{2}{*}{ Fuente } & \multirow{2}{*}{ T.A } \\
\hline & \multicolumn{2}{|c|}{ m3/ha-cosecha } & \multicolumn{2}{|c|}{ Ton/ha } & & $\mathrm{Kg} / \mathrm{m} 3$ & $\mathrm{Kg} / \mathrm{m} 3$ & & \\
\hline \multirow{4}{*}{ China } & 7100 & 8750 & 7.1 & 7.0 & 25.0 & 1.00 & 0.80 & (36) & \multirow{6}{*}{ AWD } \\
\hline & 9250 & 9857 & 7.4 & 6.9 & 14.3 & 0.80 & 0.70 & $(37)$ & \\
\hline & 11000 & 11625 & 9.9 & 9.3 & 12.5 & 0.90 & 0.80 & (38) & \\
\hline & 6875 & 7714 & 5.5 & 5.4 & 14.3 & 0.80 & 0.70 & (39) & \\
\hline Pakistán & 15235 & 14933 & 2.59 & 2.24 & 13.3 & 0.17 & 0.15 & $(40)$ & \\
\hline \multirow{8}{*}{ Japón } & 16000 & \multirow{7}{*}{$22000^{*}$} & 4.8 & \multirow{7}{*}{$4.4^{*}$} & 50.0 & 0.30 & & $(41)$ & \\
\hline & 7970 & & 9.9 & & 520.0 & 1.24 & & $(42)$ & \multirow{17}{*}{ Aspersión } \\
\hline & 8680 & & 9.6 & & 455.0 & 1.11 & & (43) & \\
\hline & 9110 & & 11.4 & & 525.0 & 1.25 & 0.20 & \multirow{5}{*}{$\left(44^{*}\right)$} & \\
\hline & 13130 & & 11.3 & & 330.0 & 0.86 & & & \\
\hline & 13010 & & 10.6 & & 305.0 & 0.81 & & & \\
\hline & 8910 & & 8.1 & & 355.0 & 0.91 & & & \\
\hline & 10750 & 19750 & 8.6 & 7.9 & 100.0 & 0.80 & 0.40 & & \\
\hline \multirow{2}{*}{ Italia } & 7459 & 15000 & 8.0 & 8.2 & 96.2 & 1.07 & 0.55 & \multirow{2}{*}{$\left(45^{*}\right)$} & \\
\hline & 5935 & 15000 & 7.2 & 8.2 & 121.9 & 1.21 & 0.55 & & \\
\hline \multirow{2}{*}{ España } & 10300 & 16000 & 5.5 & 6.6 & 29.3 & 0.53 & 0.41 & \multirow{2}{*}{$\left(46^{*}\right)$} & \\
\hline & 8720 & 16000 & 5.6 & 6.7 & 52.4 & 0.64 & 0.42 & & \\
\hline \multirow{5}{*}{ China } & 6440 & 13400 & 5.3 & 6.8 & 60.8 & 0.82 & 0.51 & \multirow{2}{*}{ (15) } & \\
\hline & 6120 & 13610 & 5.8 & 7.9 & 63.8 & 0.95 & 0.58 & & \\
\hline & 3750 & \multirow{2}{*}{15000} & 6.0 & 7.5 & 220.0 & 1.60 & \multirow{2}{*}{0.50} & \multirow{2}{*}{$(47)$} & \\
\hline & 5778 & & 5.2 & 7.5 & 80.0 & 0.90 & & & \\
\hline & 14750 & 42500 & 5.9 & 8.5 & 100.0 & 0.40 & 0.20 & $(12)$ & \\
\hline Malasia & 5526 & 15385 & 3.15 & 6.0 & 46.2 & 0.57 & 0.39 & $(48)$ & \\
\hline \multirow{11}{*}{ EUA } & 7500 & \multirow{7}{*}{$9300^{*}$} & 10.3 & & 71.3 & 1.37 & & (49) & \\
\hline & 5067 & & 6.8 & & 68.8 & 1.35 & & & \\
\hline & 5083 & & 7.3 & & 80.0 & 1.44 & & & \\
\hline & 5037 & & 8.2 & $7.4^{*}$ & 103.8 & 1.63 & 0.80 & $(50 \quad 51 *)$ & Aspersión \\
\hline & 5061 & & 7.4 & & 83.8 & 1.47 & & $(30,51)$ & \\
\hline & 5074 & & 7.5 & & 85.0 & 1.48 & & & \\
\hline & 5043 & & 7.0 & & 72.5 & 1.38 & & & \\
\hline & 6530 & 10340 & - & - & - & - & - & $(52)$ & DR \\
\hline & 7030 & 9300 & 7.7 & 7.4 & 36.3 & 1.09 & 0.80 & $(51)$ & \\
\hline & 6770 & 11170 & 12.1 & 11.4 & 74.5 & 1.78 & 1.02 & (53) & MIRI \\
\hline & 6190 & 10340 & - & - & - & - & - & $(52)$ & \\
\hline Uruguay & 12126 & 13550 & 9.3 & 8.4 & 22.6 & 0.76 & 0.62 & $(54)$ & \\
\hline & 11140 & 13760 & 8.98 & 9.25 & 20.9 & 0.81 & 0.67 & & \\
\hline Brasil & 10230 & 11710 & 9.7 & 9.98 & 11.8 & 0.95 & 0.85 & (55) & IR \\
\hline & 11450 & 14300 & 7.06 & 6.75 & 31.9 & 0.62 & 0.47 & & \\
\hline
\end{tabular}


Tabla 3. Rendimiento SICA vs el Sistema Convencional (SC) (Fuente: Autores).

\begin{tabular}{|c|c|c|c|c|c|c|c|}
\hline \multirow[t]{2}{*}{ Zona T } & $\begin{array}{c}\text { R. } \\
\text { T.A }\end{array}$ & R. SC & \multirow[t]{2}{*}{ Fuente } & \multirow[t]{2}{*}{ Zona ST } & $\begin{array}{c}\text { R. } \\
\text { T.A }\end{array}$ & $\begin{array}{l}\text { R. } \\
\text { SC }\end{array}$ & \multirow[t]{2}{*}{ Fuente } \\
\hline & \multicolumn{2}{|c|}{ Ton/ha } & & & \multicolumn{2}{|c|}{ Ton/ha } & \\
\hline \multirow{2}{*}{ India } & 5,4 & 4,6 & $(56)$ & Nepal & 5,4 & 5,5 & (69) \\
\hline & 2,88 & 2,28 & (57) & Bangladesh & 7,1 & 7,6 & (70) \\
\hline Panamá & 4,7 & 3,4 & (58) & Afganistán & 9 & 5,1 & (71) \\
\hline Brasil & 4,69 & 5,3 & $(59)$ & Irak & 7 & 4,7 & $(72)$ \\
\hline Ecuador & 8,8 & 3,8 & $(60)$ & Japón & 6,3 & 6,7 & (73) \\
\hline \multirow{3}{*}{ Madagascar } & 9.9 & 3,8 & (61) & Timor Leste & 2,94 & 3,2 & (74) \\
\hline & 5,2 & 2,6 & $(62)$ & Sri Lanka & 7,6 & 6,9 & $(75)$ \\
\hline & 10,4 & 2 & (63) & \multirow{3}{*}{ China } & 12,2 & 10 & $(76)$ \\
\hline Gambia & 7,8 & 2,5 & (64) & & 7,9 & 7,9 & $(77)$ \\
\hline Mali & 9,1 & 5,5 & $(65)$ & & 11,7 & 11,7 & $(78)$ \\
\hline \multirow{2}{*}{ Kenia } & 9,6 & 5 & $(66)$ & Indonesia** $^{* *}$ & 7,61 & 4,27 & $(79)$ \\
\hline & 7,6 & 6,2 & $(67)$ & \multirow{2}{*}{ Filipinas** } & 7,1 & 6,6 & $(80)$ \\
\hline Camboya & 2,6 & 1,9 & (68) & & 3 & 4,1 & $(81)$ \\
\hline
\end{tabular}

La tecnología $A W D$ se apoya de la inserción de la mitad de un tubo de $40 \mathrm{~cm}$ de longitud perforado y de $15 \mathrm{~cm}$ de diámetro, ubicado verticalmente en forma de batería piezométrica en el suelo para medir la altura del agua. El riego intermitente consiste en inundar el campo hasta obtener una lámina de $2 \mathrm{~cm}$ por encima de la superficie del suelo y posteriormente se deja secar hasta alcanzar una lámina de agua de $15 \mathrm{~cm}$ por debajo de la superficie. De esta forma se alternan periodos de inmersión con periodos de no inmersión durante la temporada de crecimiento $(36 ; 38)$. El primer ciclo de alternancia húmedo/seco se realiza de diez a quince días después de trasplantar y los ciclos continúan hasta el comienzo de la floración.

Se encontró que el AWD conlleva en promedio a un AA y WUE de $21.6 \%$ y $0.66 \mathrm{~kg} / \mathrm{m}^{3}$ respectivamente. Los estudios sugieren que el AWD reduce el número de horas de riego sin una reducción significativa del rendimiento, aunque se utilicen variedades de alto rendimiento desarrolladas para el sistema de riego continuo (36). Colombia busca mapear las áreas adecuadas para el uso del AWD y en complemento, el Programa de Adopción Masiva de tecnología de
FEDEARROZ promueve el cultivo con suelos bajo saturación, ya que se alcanzan rendimientos similares al arroz bajo el SC, buscando regar más área con el agua disponible. El AA y el WUE en suelos saturados en promedio son del $62 \%$ y de 1.5 $\mathrm{kg} / \mathrm{m}^{3}$, respectivamente, Tabla 1 .

En el riego corrido la distribución del agua por gravedad es controlada por diques construidos "al ojo" o a curva de nivel (WUE promedio de $0.51 \mathrm{~kg}$ / $\mathrm{m}^{3}$ ), rectos o en contornos, implicando diferentes eficiencias en el uso del agua. En la década de 1980, y de acuerdo con Smith (52), los productores de arroz en Mississippi (EUA) iniciaron con la nivelación a pendientes uniformes de 0.1 a $0.2 \%$ y se fomentó el uso de diques rectos en sustitución de diques construidos a lo largo de los contornos topográficos, lo cual ahorraba un $17 \%$ de agua aproximadamente. Posteriormente utilizaron los campos "sin pendiente", reduciendo el uso de riego en aproximadamente un 55\% con respecto al SC.

En Colombia, el arroz manejado en piscinas, arroz sembrado por trasplante en campos "sin pendiente" y con fangueo, demanda menor 
cantidad de agua y tiene menores pérdidas de suelo que el SC $\left(8958 \mathrm{~m}^{3} /\right.$ ha y 1 ton/ha-año vs $33630 \mathrm{~m}^{3}$ /ha-cosecha y 10.8 ton/ha-año; 7). No obstante las piscinas, están limitadas por la topografía, la morfología de los suelos y la inversión inicial, ya que no todas las condiciones permiten su adecuación y la mayoría de los agricultores que son arrendatarios de la tierra evitan invertir (7). El horizonte compactado que crea el fangueo degrada la estructura de los suelos para disminuir la percolación profunda entre 5 y $9 \%$ del total del agua aplicada, lo que imposibilita su recuperación para cultivos diferentes al arroz (46).

En riego corrido la labranza se orienta a generar una condición física para incrementar la infiltración y disminuir la escorrentía; sin embargo, se recomienda que sea mínima y solo en caso de compactación, se debe implementar labranza profunda con cincel vibratorio (10). En el distrito de riego del río Zulia (Norte de Santander, Colombia), donde se abastecen aproximadamente 8500 hectáreas de arroz y se cultiva en suelos con mínima pendiente $(0.3 \%)$, el promedio de la pérdida de suelo por cosecha en terreno mecanizado (120 días fase vegetativa y 88 días de riego) es de 1.1 ton/ha, y con el suelo no laboreado fue estimada en 0.15 ton/ha (82).

La implementación adecuada de la tecnología de riego a curvas de nivel y taipas para contribuir a la efectividad de la siembra, del riego, la mano de obra y los insumos, requiere de un levantamiento topográfico previo y una micro-nivelación. De esta forma la marcación de las curvas de nivel es más precisa y ahorra recursos en la preparación del suelo. Con el uso de la taipa se construyen diques (caballones) aplanados y distanciados verticalmente entre $3 \mathrm{~cm}$ y $10 \mathrm{~cm}$ para pendientes bajas y fuertes, respectivamente, con una altura aproximada de $17 \mathrm{~cm}$. Para evitar aumentar la percolación, se recomienda limitar la longitud de las melgas por medio de canales pequeńos distanciados entre ellos a $50 \mathrm{~m}, 75 \mathrm{~m}$ o $100 \mathrm{~m}$, según sea la textura del lote, ligera, media o pesada respectivamente (10).
El riego por MIRI se basa en politubos de un diámetro determinado que se extienden a lo largo del cultivo y de manera espaciada presentan orificios para la salida de un caudal establecido. El MIRI se ha combinado con los diques en contornos, diques rectos y pendientes cero (ver Figuras 4 y 5). Esto mejora la eficiencia en la conducción del agua presentando menores pérdidas por percolación, infiltración lateral y evaporación. En Uruguay, se han establecido ahorros hasta de un $50 \%$ en el volumen de agua y un $30 \%$ en costo de mano de obra (54). En Colombia se han realizado pruebas en lotes comerciales de arroz en los municipios de Montelíbano, Córdoba y Caucasia (83). De la experiencia en su implementación en el cultivo de cańa de azúcar en Colombia, se recomienda que se incluya en los criterios de selección, su vulnerabilidad al hurto y vandalismo.

\section{El riego inicial con cuatro hojas verdaderas en la} planta busca que la semilla de arroz germine con la humedad del lote, economizando así un moje (aproximadamente $450 \mathrm{~m}^{3} / \mathrm{ha}$ ). En las prácticas regionales en Colombia se identificó el uso de un riego precoz después de la siembra, con lo cual se puede destapar la semilla o no germina por falta de oxígeno, disminuyendo el porcentaje de germinación y consecuentemente se induce al uso de altas densidades de siembra $(120 \mathrm{~kg} / \mathrm{ha}$ a $220 \mathrm{~kg} / \mathrm{ha}$ ). La implementación de la técnica implica monitorear la humedad del suelo para que sea siempre la mínima requerida durante la germinación de las semillas.

Las quemas, la mecanización y el sobre-laboreo han reducido la capacidad natural del suelo para retener la humedad. Por lo tanto, Cuevas (84) realizó la evaluación de hidro-retenedores artificiales de humedad de buena estabilidad mecánica y biológica (Stockosorb), en suelos arroceros del Distrito de Riego del Río Zuila (Colombia), resaltando que la dosis de $60 \mathrm{~kg} / \mathrm{ha}$ aumentó la retención de agua en un 20\% (ver Figura 6), redujo la frecuencia de riego (45-51 días) y aumentó la producción hasta un 58\%. 


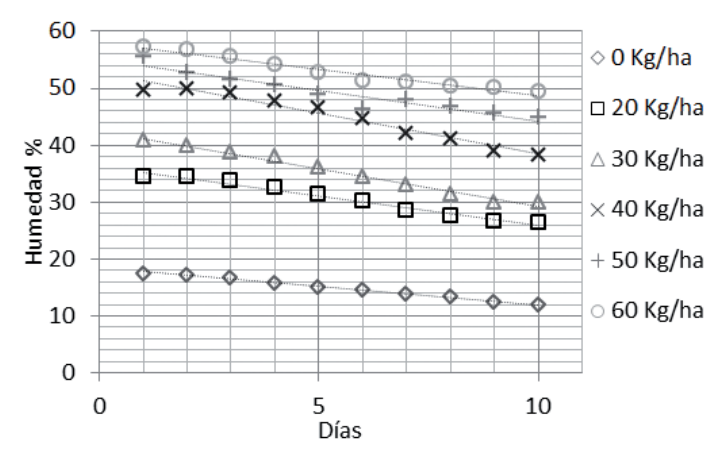

Figura 6. Retención de la humedad con diferentes dosis de Stockosorb en el suelo (Adaptado de: (84)).

Cada gramo de Stockosorb (copolímeros de acrilamida/ácido acrílico a base de sal potásica), puede absorber y retener hasta 160 gramos de agua, deshidratándose e hidratándose en el tiempo hasta unos 5 años.

Cuevas (85) recomienda realizar la fertilización nitrogenada en seco 12 horas antes del riego para su efectividad y para la disminución de gases de efecto invernadero. En el proceso de fertilización con úrea, el amonio $\left(\mathrm{NH}_{4}^{+}\right)$se fija mejor al suelo seco (carga negativa), mientras que con el suelo húmedo la interface suelos-agua favorece las velocidades de nitrificación $\left(\mathrm{NO}_{3}^{-}\right)$que no coinciden con la tasas de asimilación de la planta, por lo cual hay pérdida por desnitrificación $\left(\mathrm{N}_{2} \mathrm{O}\right)$.

\section{El potencial del arroz aeróbico}

En la Figura 5A se resalta como todas las T.A seleccionadas en la revisión, presentan una productividad del agua mayor al SC, aunque no ocurre lo mismo con los rendimientos (ver Figura 5B). Se resalta que el arroz aeróbico en el trópico no ha alcanzado la productividad del SC (arroz bajo inundación) y está por debajo de Japón y EUA. Según Kato y Katsura (1) en el subtrópico, el arroz aeróbico bajo riego ha presentado rendimientos superiores a las 8 ton/ha superando el arroz bajo inundación en: a) biomasa (en promedio 17.218.5 ton/ha vs $14.7-18.5$ ton/ha); b) eficiencia en el uso de la radiación $(1.13-1.72 \mathrm{~g} / \mathrm{MJ}$ vs 1.05 - $1.68 \mathrm{~g} / \mathrm{MJ})$; c) acumulación de nitrógeno

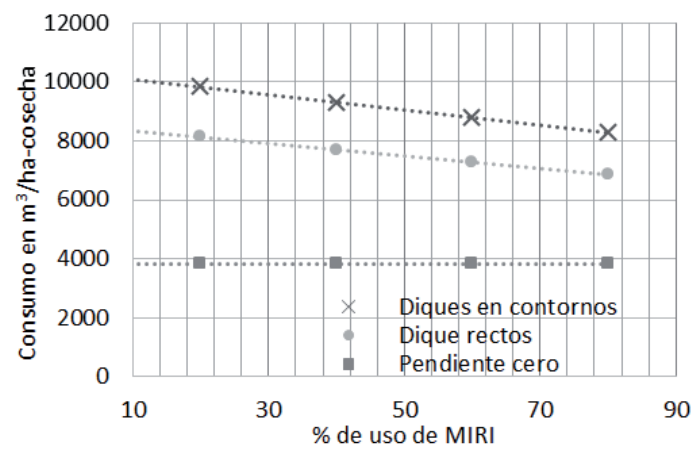

Figura 7. Agua consumida y porcentaje de uso de MIRI en arroz producido con diferentes variaciones en el riego por gravedad (Adaptado de: (52)).

en la madurez (194-233 kg N/ha vs $142-173 \mathrm{~kg}$ $\mathrm{N} / \mathrm{ha}$ ); d) número de espigas por unidad de área con el suelo cerca de capacidad de campo (4-4.4 $\mathrm{cm}^{-2}$ vs. 3.2-3.8 $\mathrm{cm}^{-2}$ ); e) eficiencia en el uso del agua $\left(1.4-2.3 \mathrm{~kg} / \mathrm{m}^{3}\right.$ vs $\left.0.9 \mathrm{~kg} / \mathrm{m}^{3}\right)$. No obstante la disponibilidad de nutrientes es más crítica en arroz aeróbico que en arroz por inundación, debido a la inmovilización de fósforo $(\mathrm{P})$ en los suelos ácidos y la volatilización del Nitrógeno $(\mathrm{N})$, además de la posibilidad de sufrir deficiencias de Hierro $(\mathrm{Fe})$, Manganeso $(\mathrm{Mn})$ y Zinc $(\mathrm{Zn})$ en suelos con $\mathrm{pH}$ mayores a 6. El arroz aeróbico está limitado en la capacidad de tomar el agua del suelo (el umbral para mantener la traspiración es de -15 a -25 Kpa en una profundidad de $20 \mathrm{~cm}$ ), debido a la longitud total de la raíz.

\section{El arroz como proveedor de servicios ecosistémicos}

El cultivo de arroz inundado es considerado como hábitat de aves acuáticas y como humedal artificial, ya que ofrece un hábitat de forrajeo y refugio contra los depredadores similar a los ecosistemas naturales, ayudando a la conservación de las especies $(86,87)$. Las aves no son una plaga para el cultivo de arroz, pues solo $10 \%$ de las aves que se encuentran en el cultivo son consumidoras del mismo y de estas solo algunas causarían un aparente daño al cultivo. Por el contrario, las aves pueden contribuir al control de plantas nocivas para el cultivo, además de alimentarse de roedores, herpetos e insectos. 
En Colombia desde el año 2009 se promueven prácticas en el cultivo para proporcionar un hábitat para las aves a través del proyecto titulado: "La alas del arroz" de la Fundación Calidris (88) y ha permitido identificar 112 especies asociadas al arroz y 12 especies playeras (10 migratorias). El nivel de agua, el periodo de inundación, el uso de pesticidas, la estructura y el tamaño de la planta de arroz, son los principales aspectos que intervienen en la composición y abundancia de especies (86).

Se destaca el papel de las cercas vivas en el cultivo o las barreras anchas de bosque, las cuales rompen el viento y propician un microclima para el establecimiento de biorreguladores tales como arańas, insectos $\mathrm{y}$ aves que sirven para el control biológico. Los árboles influyen en el ciclo del agua incrementando la intercepción de la lluvia, la transpiración y la retención de agua en el suelo, de tal manera que se reduce la escorrentía, aumenta la infiltración y reduce la erosión. Se destaca además que los árboles contribuyen a la producción de leña, forraje, frutas y aumentan la complejidad estructural y florística del paisaje (89). En un estudio realizado por Alam (90) para evaluar la incidencia de enfermedades del cultivo de arroz en presencia simultánea con especies arbóreas se encontró que aquellos árboles que evitan la penetración de la luz tendrán una mayor relación con enfermedades en el cultivo.

La rizipiscicultura es considerada dentro de los sistemas ingeniosos del patrimonio agrícola mundial (91) y representa un ingreso económico adicional para los agricultores con sostenibilidad ambiental y alimentaria. Existe un beneficio mutuo entre el arroz, los peces y microorganismos acuáticos que optimizan cada subsistema. Algunas características de la "Rizipiscicultura" son: a) la restricción en el uso de pesticidas y plaguicidas para evitar la muerte de los peces y b) el consumo de agua puede ser menor que con las dos prácticas por separado. En el departamento de Córdoba (Colombia), se ha utilizado la carpa capim (Ctenopharyngodon idella), carpa plateada (Hypophthalmicthys molitrix), cachama (Colossoma macropomum), tilapia nilótica (Oreochromis niloticus) y bocachico (Prochilodus magdalenae), con buenos rendimientos de arroz (92).

\section{Reflexión para Colombia}

El uso eficiente del agua determina el futuro del cultivo de arroz en Colombia, por su relación con factores como el área cosechada, el rendimiento, los costos de producción y la sostenibilidad en el tiempo. No obstante hay un evidente rezago en la adopción de tecnologías y al tomar como referencia el caso de los agricultores en Misisipi (EUA), la brecha cultural en el ahorro de agua es muy grande. Por lo tanto, se debe trabajar en una mejor comprensión de los mecanismos que son la base de la adopción tecnológica y aunar esfuerzos para reforzar el cambio hacia un uso racional del agua.

Como medidas de mitigación de las pérdidas de suelo y agua en el riego corrido es necesario iniciar por prácticas básicas tales como evitar los flujos preferenciales, utilizar disipadores de velocidad, valorar las barreras naturales, garantizar la calidad de la nivelación del terreno con el adecuado uso de las tecnologías, implementar aliviaderos y politubos para la distribución del agua; así como trabajar en el cambio de cultura frente al uso excesivo de agua, donde se busca cultivar bajo suelo saturado o con intermitencia en el riego. Lo anterior permitirá reducir la sedimentación en las estructuras, canales de riego y drenajes, reducir la contaminación del agua, aumentar la rentabilidad del cultivo y el área sembrada con riego. Es importante capturar y hacer uso in situ del agua lluvia, que ayudado por un tanque artesanal (previa calibración) o el acceso a la red de estaciones meteorológicas, permite determinar cuánto aporta la precipitación, cuanto se evapora y así cuándo es necesario regar. Además, las variables meteorológicas (temperatura, radiación solar, precipitación, etc.) y el uso de modelos agroclimáticos permiten establecer las fechas de siembra más adecuadas. 
Para la disponibilidad de agua, en Costa Rica, México y Nicaragua ha sido analizada la cosecha de agua entre el 2008 y el 2012 por el CIAT y el Fondo Latinoamericano para Arroz de Riego (FLAR), logrando rendimientos de 9.5 ton/ha (grano limpio y seco), demostrando que los costos de construcción de un reservorio pueden ser amortizados aproximadamente en 5 años y después de 10 ańos, el costo de $\mathrm{m}^{3}$ es despreciable (93). En Colombia, para sostener un cultivo de arroz se necesita cerca de $900 \mathrm{~mm}$ de lluvia acumulada, cantidad superada por la precipitación en ciertas zonas arroceras, por lo tanto se hace necesaria la construcción y el mantenimiento de reservorios, sin olvidar adoptar las tecnologías para el ahorro de agua como base para la explotación racional y sostenible, ya que el volumen de agua que se requiere almacenar varía de forma creciente con el agua demandada por el cultivo, el número de hectáreas a regar y las pérdidas del sistema de riego.

Se debe priorizar en la medición efectiva del consumo de agua y en la cantidad de vertimientos (indispensable para controlar su uso), planear, dirigir, entender o emprender acciones de mejora por parte de los usuarios e instituciones. Los medidores de caudal con registro continuo son considerados en los instrumentos económicos y financieros de la gestión ambiental colombiana (94), ya que el volumen es la base para el cobro. El valor a pagar (VP) por la Tasa por Uso de Agua (TUA) fue modificado por el Decreto Nacional 4742 de 2005 y resulta del producto de la TUA, expresada en pesos $/ \mathrm{m}^{3}$, el volumen $\mathrm{m}^{3}$ captado (V) y el factor de costo de oportunidad $\left(\mathrm{F}_{\mathrm{OP}}\right)$. En los casos donde el sujeto pasivo no presente los reportes de volumen de agua captada, el cobro se realizará por el caudal concesionado; es decir, cobrando como si se captara y utilizara agua continuamente y sin interrupción. La medición continua y el reporte ha permitido en algunos casos aislados en Colombia, una disminución promedio del 54\% del valor a pagar y una ahorro del $17 \%$ en los volúmenes efectivamente captados en relación a los caudales concesionados (95).

Distritos de riego como ASORECIO, USOCOELLO y USOSALDAÑA (Tolima) han tenido experiencias poco exitosas en cuanto al uso de medidores de caudal, debido al rechazo generalizado de los usuarios, quienes terminaron por destruir las estructuras al poco tiempo de su instalación (96), debido a razones de tipo social y cultural. De esta forma, generalmente la facturación se basa en la estimación del flujo a través de una compuerta y el tiempo durante el cual ésta permanezca abierta, lo cual es monitoreado por un representante del distrito de riego. Sin embargo, en el canal de drenaje no se mide la cantidad ni la calidad del agua para implementar las Tasas Retributivas y Compensatorias (TRC), que buscan contribuir al control de la contaminación hídrica en el país (Decreto 2667 de 2012).

En un estudio en el Tolima realizado por Bustos $(97,98)$, se comprobó que los herbicidas glifosato, atrazina, los fungicidas carboxín, carbendazim y epoxiconazol, el antioxidante BHT, el BHTCHO, el plastificante N-BBSA y la benzofenona están llegando a los cuerpos de agua superficiales que reciben los drenajes de los cultivos de arroz, muy probablemente ligados al material en suspensión que se transporta por escorrentía. La lixiviación de glifosato y su acumulación en los sedimentos recientes de las quebradas, generan riesgos ambientales que afectan el ecosistema de la zona.

En la revisión, se encontró que la eficiencia del uso del agua $\left(\mathrm{kg} / \mathrm{m}^{3}\right)$ con los nuevos avances tecnológicos puede ser de hasta $2 \mathrm{~kg} / \mathrm{m}^{3}$, comparable con la del resto de cereales y superior a la eficiencia del SC. Se resalta el arroz aeróbico de regadío como una tecnología potencial para reducir el uso de agua. El éxito de esta tecnología depende del riego oportuno, el control la mala hierba, del desarrollo de variedades con buena conductividad hidráulica radicular y capacidad de absorber agua, lo cual es proporcional a la longitud de la raíz y su área de superficie.

\section{Agradecimientos}

Este trabajo fue realizado dentro de un proyecto financiado por el sistema de investigación, desarrollo tecnológico e innovación del SENA, SENNOVA. 


\section{Referencias}

1. Kato Y, Katsura K. Rice adaptation to aerobic soils: physiological considerations and implications for agronomy. Plant Production Sc. 2014; 17(1): 1-12.

2. Barona E. Importancia de la semilla de arroz. Arroz. 2010; 58(489): 15-21.

3. Liu M, Lin S, Dannenmann M, Tao Y, Saiz G, Zuo, K, et al. Do water-saving ground cover rice production systems increase grainyields at regional scales?. Field crops re. $2013 \mathrm{Jul}$; 150: 19-28.

4. González M., Saldarriaga G, Jaramillo O. Estimación de la demanda de agua: Conceptualización y dimensionamiento de la demanda hídrica sectorial. Estudio Nacional del Agua, Capítulo 5. IDEAM, Colombia; 2010.

5. Cortés B. C. Uso del modelo aquacrop para estimar rendimientos para el cultivo de arroz en los departamentos de Tolima y Meta. Informe del proyecto de cooperación técnica TCP/COL/3302.FAO; 2013.

6. CORTOLIMA. Plan de Gestión Ambiental Regional-PGAR 2013-2023. Corporación Autónoma Regional del Tolima; 2013.

7. Salive A, Riobueno C, Castilla L. Mejoramiento del sistema de manejo de riego en arroz. Arroz. 2004; 52 (452): $32-41$.

8. FEDEARROZ. Área, producción y rendimientos. [Internet]; 2015 [acceso 15 nov 2015]. Disponible en: http://www. fedearroz.com.co/new/apr_public.php

9. FAOSTAT data. Agriculture and Food Trade. [acceso 08 de oct 2015]. Disponible en: http://faostat 3.fao.org/home/index. html.

10. FEDEARROZ. "Manejo integrado del cultivo de arroz". Fondo Nacional del Arroz. Produmedios. Colombia; 2004.

11. Acosta, J. Evaluación del sistema intensivo de cultivo arrocero en el municipio de Purificación Tolima [Tesis pregrado]. Colombia, U. de Cundinamarca; 2011.

12. Tao H, Brueck H, Dittert K, Kreye C, Lin S, Sattelmacher B. Growth and yield formation of rice in the water-saving ground cover rice production system. Field Crops Re. 2006; 95:1-12.

13. Tao Y, Zhang Y, Jin X, Saiz G, Jing R, Guo L. More rice with less water-evaluation of yield and resource use efficiency in ground cover rice production system with transplanting. European J. of Agronomy. 2015; 68: 13-21.

14. Lampayan R, Samoy K, Sibayan E, Ella B, Jayag P, et al. Effects of alternate wetting and drying (AWD) threshold level and plant seedling age on crop performance, water input, and water productivity of transplanted rice in Central Luzon, Philippines. Paddy and Water Environment. 2015; 13(3): 215-227.

15. Lampayan R, Bouman B. Management strategies for saving water and increase its productivity in lowland rice-based ecosystems. SUMAPOL, Hangzhou, Zhejiang Province, China; 2005.

16. Martín Y, Soto F, Rodríguez YE, Morejón R. El sistema intensivo de cultivo del arroz (SICA) disminuye la cantidad de semillas para la siembra, aumenta los rendimientos agrícolas y ahorra el agua de riego. C. Tropicales. 2010; 31 (1):70-73.
17. Guerra H, Chinea E, Vidal R, Hernández N, Moreno G. Determinación de los consumos de agua en condiciones de producción, en el sistema arrocero Jesús Suárez Gayol del CAI arrocero Ruta Invasora. [acceso 15 Nov 2015]. Disponible en: http://www.cubasolar.cu/biblioteca/ecosolar/Ecosolar47/ HTML/articulo04N.htm

18. Maqueira L, Torres W, Díaz G, Torres K. Efecto del sistema intensivo del cultivo arrocero sobre algunas variables del crecimiento y el rendimiento en una variedad de ciclo corto. C. Tropicales. 2007; 28(2): 59-61.

19. Pineda. D. Aplicación de la tecnología para manejo eficiente de agua para riego. Arroz. 2010; 58 (485): 22-32.

20. Nyamai M; Mati B, Home P, Odongo B, Wanjogu R, Thuranira EG. Improving land and water productivity in basin rice cultivation in Kenya through System of Rice Intensification (SRI). Agric Eng Int: CIGR Journal. 2012 May; 14(2): 1-13.

21. Rajeshwar M, Aariff M. Comparison of System of Rice Intensification (SRI) and conventional method of rice planting under nagarjuna sagar project left canal command área of Andhra Pradesh, India. J. of Soil Science. 2008; 3(1): 53-57.

22. Thakur A, Rath S, Patil D, Kumar A. Effects on rice plant morphology and physiology of water and associated management practices of the system of rice intensification and their implications for crop performance. Paddy Water Environment. 2011; 9:13-24

23. Veeraputhiranl R, Balasubramanian R, Pandian B, Chelladurai $\mathrm{M}$, Tamilselvi R, Renganathan VG. Influence of system of rice intensification on yield, water use and economics through farmers participatory approach. Madras Agric. J. 2012 Jun; 99(4-6): 251-254.

24. Satyanarayana A, Thiyagarajan T. Opportunities for water saving with higher yield from the system of rice intensification. Irrigation Sci. 2007; 25:99-115.

25. Gopalakrishnan S, Kumar R, Humayun P, Srinivas V, Kumari BR, Vijayabharathi R, et al. Assessment of different methods of rice cultivation affecting growth parameters, soil chemical, biological, and microbiological properties, water saving, and grain yield in rice-rice system. Paddy Water Environment. 2014; 12:79-87.

26. Manjunatha B, Basavarajappa R, Pujari B. Effect of age of seedlings on growth, yield and water requirement by different system of rice intensification. Karnataka J. Agric. Sci. 2010; 23 (2): 231-234.

27. Naik BB, Reddy DR, Sreenivas G, Rani PL. Yield and Water Productivity of Aerobic Rice (Oryza sativa L.) as Influenced by Dates of Sowing and Varieties during kharif season. Journal of Rice Research. 2015; 8(1): 52-56.

28. Kadiyala M, Mylavarapu R, Li Y, Reddy G, Reddy M. Impact of aerobic rice cultivation on growth, yield, and water productivity of rice-maize rotation in semiarid tropics. Agronomy J. 2012; 104(6).

29. Bouman B, Peng S, Castañeda A, Visperas R. Yield and water use of irrigated tropical aerobic rice systems. Agric. water management. 2005; 74: 87-105.

30. Crusciol C, Arf O, Soratto R, Machado J. Extração de macronutrientes pelo arroz de terras altas sob diferentes níveis de irrigação por asperazo e de adubação. R. Brasilera Agrociência. 2003; 9: 145-150. 
31. Avasthe R, Verma S, Kumar A, Rahman H. Performance of rice (Oryza sativa) varieties at different spacing under system of rice intensification (SRI) in mid hill acid soils of Sikkim Himalayas. Indian J. of Agronomy. 2012; 57:32-37.

32. Suryavanshi P, Singh Y, Prasanna R, Bhatia A, Shivay Y. Pattern of methane emission and water productivity under different methods of rice crop establishment. Paddy Water Environment. 2013; 11:321-329.

33. Hameed K, Mosa K, Jaber, F. Irrigation water reduction using system of rice intensification compared with conventional cultivation methods in Iraq. Paddy and Water Environment. 2011; 9: 121-127.

34. Zhao L, Wu L, Li Y, Lu X, Zhu D, Uphoff, N. Influence of the system of rice intensification on rice yield and nitrogen and water use efficiency with different $\mathrm{N}$ application rates. Expl Agric. 2009; 45: 275-286

35. Zhao L, Wu L, Li Y, Animesh S, Zhu D, Uphoff N. Comparisons of yield, water use efficiency, and soil microbial biomass as affected by the system of rice intensification. C. in soil science and plant analysis. 2010; 41:1-12.

36. Yao F, Huang J, Cui K, Nie L, Xiang J, Liu X, et al. Agronomic performance of high-yielding rice variety grown under alternate wetting and drying irrigation. Field crops research. 2012; 126: 16-22.

37. Ye Y, Liang X, Chen Y, Liu J, Gu J, Guo R, et al. Alternate wetting and drying irrigation and controlled-release nitrogen fertilizer in late-season rice. Effects on dry matter accumulation, yield, water and nitrogen use. Field Crops Re. 2013; (144): 212-224

38. Liu L, Chen T, Wang Z, Zhang H, Yang J, Zhang J, et al. Combination of site-specific nitrogen management and alternate wetting and drying irrigation increases grain yield and nitrogen and water use efficiency in super rice. Field crops re. $2013 ; 154: 226-235$.

39. Cabangon R, Tuong T, Castillo E, Bao L, Lu G, Wang G, et al. Effect of irrigation method and $\mathrm{N}$-fertilizer management on rice yield, water productivity and nutrient-use efficiencies in typical lowland rice conditions in China. Rice Field Water Environ. 2004; 2:195-206.

40. Rehman H, Kamran M, Basra S, Afzal I, Farooq M. Influence of seed priming on performance and water productivity of direct seeded rice in alternating wetting and drying. Rice Science. 2015; 22(4): 189-196.

41. Matsuo N, Mochizuki T. Growth and yield of six rice cultivars under three water-saving cultivations. Plant Prod. Sci. 2009; 12: $514-525$.

42. Katsura K, Nakaide Y. Factors that determine grain weight in rice under high-yielding aerobic culture: the importance of husk size. Field Crops Res. 2011; 123: 266-272.

43. Okami M, Kato Y, Yamagishi J. Grain yield and leaf area growth of direct-seeded rice on flooded and aerobic soils in Japan. Plant Prod. Sci. 2013, 16: 276-279.

44. Kato Y, Okami M, Katsura K. Yield potential and water use efficency of aerobic rice in Japan. Field crops research. 2009; 113: 328-334.

45. Spanu A, Murtas A. Water use and crop coefficients in sprinkler irrigated rice. Ital. J. Agron. / Riv. Agron. 2009; 2:47-58
46. Blanco A. Agronomía del cultivo del arroz en riego por aspersión: variedades, riego, fertilización y control de malas hierbas [Tesis doctoral]. España, U. de Lleida; 2014.

47. Huaqi W, Bouman BAM, Zhao D, Changgui W, Moya PF. Aerobic rice in northern China: opportunities and challenges. En: Water-wise rice production. Proceedings of the International Workshop on Water-Wise Rice Production. (Eds. Bouman BAM, Hengsdijk H, Hardy B, Bindraban PS, Tuong TP, Ladha JK). Los Baños, Philippines. Los Baños (Philippines): International Rice Research Institute; 2002.

48. Chan C, Zainudin H, Saad A, Azmi M. Productive water use in aerobic rice cultivation. J. Trop. Agric and Fd. 2012; 49(1): 117-126

49. Stevens G, Vories E, Heiser J, Rhine M. Experimentation on cultivation of rice irrigated with a center pivot system. In T.S. Lee ed., Irrigation systems and practices in challenging environments. InTech. 2012: 233-254.

50. Vories E, Stevens W, Tacker P, Griffin T, Counce P. Rice production with center pivot irrigation. Applied engineering in agriculture. 2013; 29(1): 51-60.

51. Vories ED, Tacker PR, Hogan R. Multiple-inlet approach to reduce water requirements for rice production. Am. Soc. Agric. Eng. 2005; 21: 611-616.

52. Smith MC. Water use estimates for various rice production systems in Mississippi and Arkansas. Irrig. Sci. 2007; 25:141-147.

53. Massey J, Walker T, Anders M, Smith M, Avila LA. Farmer adaptation of intermittent flooding using multiple-inlet rice irrigation in Mississippi. Agricultural Water Management. 2014; 146: 297-304.

54. Bandeira S. y Böcking B. Riego de arroz por mangas; 2015 [citado 15 Nov 2015]. Disponible en: http://www. acpaarrozcorrientes.org.ar/Informacion_de_Interes/Riego_ de_Arroz_por_Mangas_2014.pdf

55. Avila L, Martini L, Mezzomo R, Refatti JP, Campos R, Cezimbra $\mathrm{DM}$, et al. Rice water use efficiency and yield under continuous and intermittent Irrigation. J. of Agron. 2015; 107:442-448.

56. Adusumilli R, Laxmi, S. Potential of the system of rice intensification for systemic improvement in rice production and water use: the case of Andhra Pradesh. Paddy Water Environment. 2011; 9:89-97.

57. Krishna A, Biradarpatil N, Channappagoudar B. Influence of system of rice intensification cultivation on seed yield and quality. Karnataka J. Agric. Sci. 2008; 21(3):369-372.

58. Turmel M, Espinosa J, Franco L, Pérez C, Hernández H, González E, et al. On-farm evaluation of a low-input rice production system in Panama. Paddy Water Environment. 2011; 9:155-161.

59. Gehring C, Gomes E, Boddey R. System of rice intensification (SRI) in southeastern lowlands of Amazonia a viable alternative for smallholder irrigated rice production?; 2008 [citado 12 Jul 2015]. Disponible en: http://www.tropentag.de/2008/ abstracts/full/40.pdf.

60. Chang J. Breve reseña del Sistema Intensificado del Cultivo de Arroz SICA primera validación para pequeños productores en Ecuador; 2008 [citado 15 Jul 2015]. Disponible en:

61. http://sri.ciifad.cornell.edu/countries/ecuador/ EcuGilLibroCultivodiArroz08.pdf. 
62. Tsujimoto Y, Horie T, Randriamihary H, Shiraiwa T, Homma k. Soil management: The key factors for higher productivity in the fields utilizing the system of rice intensification (SRI) in the central highland of Madagascar. Agric Syst. 2009; 100: 61-71

63. Barison J, Uphoff N. Rice yield and its relation to root growth and nutrient-use efficiency under SRI and conventional cultivation: an evaluation in Madagascar. Paddy Water Environment. 2011; 9:65-78.

64. Uphoff N, Randriamiharisoa R. Reducing water use in irrigated rice production with the Madagascar System of Rice Intensification. In: Bouman BAM, et al. (Eds.), P. WaterWise Rice Production: 8- 11 April 2002, IRRI, Los Banos, Philippines; 2002. p. 356.

65. Ceesay M. An opportunity for increasing factor productivity for rice cultivation in The Gambia through SRI. Paddy Water Environ. 2013; 9:129-135.

66. Styger E, Attaher M, Guindo H, Ibrahim H, Diaty M, Abba, I, et al. Application of system of rice intensification practices in the arid environment of the Timbuktu region in Mali. Paddy Water Environment. 2011; 9:137-144.

67. Mati B, Wanjogu R, Odongo B. Introduction of the System of Rice Intensification in Kenya: experiences from Mwea Irrigation Scheme. Paddy Water Environ. 2011; 9:145-154.

68. Ndiiri J, Mati B, Home P, Odongo B, Uphoff N. Comparison of water savings of paddy rice under System of Rice Intensification (SRI) growing rice in Mwea, Kenya. J of Current Research and Review. 2012; 4(6): 63-73.

69. Ly P, Stoumann L, Bech T, Rutz D, de Neergaard A. The system of rice intensification: Adapted practices, reported outcomes and their relevance in Cambodia. Agricultural Systems. 2012; 113: 16-27.

70. Evans C, Justice S, Shrestha S. Experience with the system of rice intensification in Nepal. Cornell I; 2002. [citado 12 Nov 2015]. Disponible en: http://ciifad.cornell.edu/sri; 607-2550831; ciifad@cornell.edu.

71. Latif M, Islam M, Ali M, Saleque M. Validation of the system of rice intensification (SRI) in Bangladesh. Field Crops Re. 2005; 93: 281-292.

72. Thomas V, Ramzi A. SRI contributions to rice production dealing with water management constraints in northeastern Afghanistan. Paddy Water Environment. 2011; 9:101-109.

73. Hameed K, Jaber F, Hadi A, Elewi H, Uphoff N. Influence of system of rice intensification (SRI) methods on productivity and yield components of jasmine rice variety in Al-Muthanna province, Iraq season 2008; 2008 [citado 20 Nov 2015]. Disponible en: http://sri.ciifad.cornell.edu/countries/iraq/ IraqAlMuthanna_JasmineRiceExper08.pdf.

74. Chapagain T, Riseman A, Yamaji E. Assessment of system of rice intensification (SRI) and conventional practices under organic and inorganic management in Japan. Rice Science. 2011; 18(4): 311-320.

75. Noltze M, Schwarze S, Qaim M. Impacts of natural resource management technologies on agricultural yield and household income: The system of rice intensification in Timor Leste. Ecological Economics. 2013; 85: 59-68.
76. Nissanka S, Bandara T. Comparison of productivity of system of rice intensification and conventional rice farming systems in the dry-zone region of Sri Lanka. Fourth I. Crop Sci. Congress, Queensland, Australia. 2004: 1177.

77. Shengfu A, Xiehui W, Zhongjiong X, Shixiu X, Chenquan L, Yangchang L. Assessment of using SRI with the super hybrid rice variety Liangyoupei. Uphoff, et al. (Eds.), Assessments of the System of Rice Intensification (SRI): Proceedings of CIIFAD, Sanya, China, Ithaca, NY, USA; 2002. pp. 112-1115.

78. Sheehy J, Penga S, Dobermann A, Mitchell P, Ferrer A, Yang J, et al. Fantastic yields in the system of rice intensification: fact or fallacy?. Field Crops Re. 2004; 88: 1-8.

79. Qingquan, Y. The system of rice intensification and its use with hybrid rice varieties in China. In: Uphoff, et al. (Eds.), Assessments of the System of Rice Intensification (SRI): Proceedings of an International Conference, Sanya, China, CIIFAD, Ithaca, NY, USA; 2002. pp. 109-111.

80. Hasan M, Sato S. Water saving for paddy cultivation under the system of rice intensification in eastern Indonesia. J. Tanah Lingk. 2007; 9 (2):57-62

81. Markarim A, Balasubramanian V, Zaini Z, Syamsiah I, Diratmadja I, Arafah H, et al. System of rice intensification (SRI): evaluation of seedling age and selected components. In: Bouman, B.A.M., et al. (Eds.), P. Water-Wise Rice Production: IRRI, Los Banos, Philippines; 2002. p. 356.

82. Rickman, J.F. Preliminary results: rice production and the system of rice intensification; 2004. [citado 25 Nov 2015]. Disponible en: http://ciifad.cornell.edu/sri/countries/philippines/irrieval.pdf.

83. Cuevas M A. Perdidas por escorrentía en suelos arroceros del distrito de riego del rio Zulia. Arroz. 2012; 60 (501).

84. Perez C. Los politubos alternativa para el manejo de agua en cultivo de arroz en Colombia. Arroz. 2015; 63(515): 33 -39.

85. Cuevas M, Ovalle P. Evaluación de hidro-retenedores de humedad en suelos arroceros, en el distrito de riego del Rio Zuila. Arroz. 2004; 52 (450): 4-10.

86. Cuevas M A. Tiempo de suministro del riego sobre la eficiencia de la fertilización nitrogenada y la velocidad de nitrificación de la úrea en el desarrollo de fedearroz 2000. Arroz. 2014; 62(511): 36-42.

87. Elphick S, Baicich P, Parsons K, Fasola M, Mugica L. The future for research on waterbirds in rice fields. Waterbird. 2010; 33: 231-243.

88. Acosta M, Mugica L, Blanco D, Bernabé L, Antunes R, et al. Birds of rice fields in the Americas. Waterbirds. 2010; 33(1): 105-122.

89. Cifuentes S, Suarez J, Castillo L. Manejo integrado de plagas en cultivos de arroz: una práctica amigable con el ambiente; 2012. [citado 22 Jul 2015]. Asociación Calidris. Disponible en: http://calidris.org.co/?p=2149.

90. Asase A, Tetteh DA. The role of complex agroforestry systems in the conservation of forest tree diversity and structure in southeastern Ghana. En: Agroforest system. 2010; 79:335-368.

91. Alam A, Khalequzzaman K, Anam M, Islam N, Rahman G. Effect of tree rice associations and orientations on the incidence of major diseases of rice. J of biological sc. 2002; 2(7): 492-493. 
92. Parviz K.y Altieri M. Un Legado para el Futuro, Sistemas Ingeniosos del Patrimonio Agricola Mundial. ONU, Roma; 2011.

93. Perez R. Producción ecológica de arroz con peces "Rizipiscicultura". Arroz. 2005; 53(458): 13 - 20.

94. Jaramillo S, Pulver E, Moreira S. Transformación de una agricultura de secano a riego a través de la cosecha de agua en Costa Rica, México, Nicaragua. XII C. I. do arroz para America Latina e Caribe. Porto alegre, Brasil; 2015.

95. Decreto 155 de 2004, por el cual se reglamenta el artículo 43 de la Ley 99 de 1993 sobre tasas por utilización de aguas y se adoptan otras disposiciones. Ministerio de Ambiente. Diario Oficial No. 45.439 (enero 23 de 2004).
96. CORNARE. Informe Tasas por Utilización del Recurso Hídrico. El Santuario Antioquia; 2013 [citado 12 Jul 2015]. Disponible en: http://200.30.82.162/instrumentoseconomicos/tasa-por-uso/Informes/Tasas-por-Uso-2013.pdf

97. Urrutia, N. Sustainable management after irrigation system transfer. Experiences in Colombia. [Phd thesis]. Netherlands, Delft; 2006.

98. Corrales LC, Romero DMA, Macías JAB, Vargas AMC. Bacterias anaerobias: procesos que realizan y contribuyen a la sostenibilidad de la vida en el planeta. Nova. 2015;13(24):55-82. 